\title{
Impacto da educação na saúde de pacientes com artrite reumatoide - estudo piloto
}

\section{Impact of education on rheumatoid arthritis patients' health - pilot study}

\author{
Lorena Costa Duval Borges ${ }^{1}$, Lidia Assis Chaves $\mathbf{1}$, Júnia Amorim Andrade ${ }^{2}$, \\ Luiza Cassimiro Silva ${ }^{2}$, Ana Letícia Fontes de Oliveira Marcelino², \\ Rosalma Aparecida Chaves ${ }^{2}$, Adriana Maria Kakehasi ${ }^{3}$, \\ Débora Cerqueira Calderaro ${ }^{3}$
}

http://dx.doi.org/10.11606/issn.2238-6149.v31i1-3p54-59

Borges LCD, Chaves LA, Andrade JA, Silva LC, Marcelino ALFO, Chaves RA, Kakehasi AM, Calderaro DC. Impacto da educação na saúde de pacientes com artrite reumatoide - Estudo Piloto. Rev Ter Ocup Univ São Paulo. 2020 jan.-dez.;31(1-3):54-9.

RESUMO: Objetivo: Grupos de orientação para pacientes com artrite reumatoide (AR) têm boa aceitação, mas benefícios controversos no tratamento dessa doença. Este estudo visa a avaliar seu impacto sobre a qualidade de vida e a dor em pacientes com AR. Métodos: Ensaio clínico piloto de intervenção em grupos de orientação que incluiu 15 pacientes com AR. Foram realizados 12 encontros de duas horas por semanas, que abordaram ações educativas sobre a doença, o tratamento, medidas de proteção articular e conservação de energia, atividades terapêuticas ocupacionais e oficinas de vivências. Foram utilizados a escala visual analógica de dor (EVA dor) e o sistema descritivo em três níveis de cinco dimensões do EuroQol e sua escala visual analógica (EQ-5D-3L e EQ-VAS) para avaliação da qualidade de vida. Resultados: Catorze (93\%) pacientes eram do gênero feminino, com idade média 61,27 anos, tempo médio de doença de 19,93 anos. Treze $(87 \%)$ deles apresentavam deformidades articulares em mãos pela AR. Houve melhora significativa no domínio cuidados pessoais do EQ-5D-3L $(\mathrm{p}=0,046)$ e tendência à redução da dor (EVA dor) e melhora da qualidade de vida (EQ-5D-3L e EQVAS), sem significância estatística $(\mathrm{P}>0,05)$. Conclusões: Apesar do pequeno número de pacientes incluídos neste estudo piloto, a melhora dos cuidados pessoais, da autopercepção da dor e da qualidade de vida dos pacientes sugere que houve impacto positivo da educação, das atividades terapêuticas ocupacionais e do compartilhamento de vivências entre os pacientes com AR.

DESCRITORES: Artrite reumatoide; Terapia ocupacional; Educação; Qualidade de vida; Dor.
Borges LCD, Chaves LA, Andrade JA, Silva LC, Marcelino ALFO, Chaves RA, Kakehasi AM, Calderaro DC. Impact of education on rheumatoid arthritis patients' health - Pilot Study. Rev Ter Ocup Univ São Paulo. 2020 Jan-Dec;31(1-3):54-9.

ABSTRACT: Background: Educational groups of patients with rheumatoid arthritis (RA) have good acceptance, but controversial benefits for its treatment. This study aims to evaluate quality of life and pain in RA patient attending education groups. Methods: Pilot clinical intervention study of educational groups including $15 \mathrm{RA}$ patients. Twelve weekly appointments lasting two hours each were held. They addressed educational actions on the disease treatment, joint protection and energy conservation measures, occupational therapeutic activities and workshops on sharing experiences. The pain visual analogue scale (VAS-pain) and the 5 domain-threedimensional descriptive system of EuroQol (EQ-5D-3L) and its visual analogue scale (EQ-VAS) were applied to assess pain and quality of life. Results: Fourteen (93\%) patients were female, with a mean age of 61,27 years, mean disease time of 19,93 years. Thirteen $(87 \%)$ of them had hand joint deformities due to RA. There was a significant improvement in the personal care domain of the EQ-5D-3L $(\mathrm{P}=0,046)$ and a tendency to pain reduction (VAS-pain) and improvement of quality of life (EQ-5D-3L and EQVAS index), without statistical significance $(\mathrm{P}>0.05)$. Conclusion: Despite the evaluation of few RA patients, the improvement of patients' personal care, pain self-perception and quality of life suggests that there was a positive impact of education, occupational therapy interventions and sharing experiences among patients with RA.

KEYWORDS: Arthritis, rheumatoid; Occupational therapy; Education; Quality of life; Pain.

1. Faculdade de Medicina do Centro Universitário de Belo Horizonte, Belo Horizonte, MG. ORCID: Borges LCD - http:/orcid.org/00000003-1156-5771; Chaves LA - http://orcid.org/0000-0001-6725-9621.E-mail: lorena.borges.med@gmail.com; lidiachavesbh@gmail.com.

2. Faculdade de Terapia Ocupacional da Universidade Federal de Minas Gerais, Belo Horizonte, MG. ORCID: Andrade JA https://orcid.org/0000-0001-7714-5214; Silva LC - http://orcid.org/0000-0002-6843-293X; Marcelino ALFO - http://orcid.org/00000001-9597-6601; Chaves RA - http://orcid.org/0000-0001-8689-3628.E-mail: amorim.junia@gmail.com; luizacassimiro.s@gmail.com; analeticiafonteso@gmail.com, rosalmachaves@hotmail.com.

3. Departamento do Aparelho Locomotor da Faculdade de Medicina da Universidade Federal de Minas Gerais, Belo Horizonte, MG. ORCID: Kakehasi AM - https://orcid.org/0000-0001-9411-7493; Calderaro DC - http://orcid.org/0000-0001-5535-3223. E-mail: amkakehasi@gmail.com; dccalderaro@gmail.com.

Endereço de correspondência: Rua Carlos Turner, 111 Bloco 4,Apart. 402, Silveira. Belo Horizonte, Minas Gerais. CEP: 31140520. E-mail: lidiachavesbh@gmail.com. 


\section{INTRODUÇÃO}

$\mathrm{A}$ artrite reumatoide (AR) é uma doença autoimune, sistêmica caracterizada por sinovite periférica crônica e por manifestações extra-articulares. Pode levar à diminuição da independência funcional, da capacidade laborativa e da qualidade de vida, em parte devido à dor e às deformidades articulares ${ }^{1,2}$.

$\mathrm{O}$ tratamento com medicamentos modificadores do curso da doença (MMCD) sintéticos ou biológicos permite diminuir ou mesmo interromper a progressão da AR. Ainda assim, o controle do acometimento articular envolve a educação do paciente e terapias que visem a auxiliar na realização das atividades motoras, uma vez que frequentemente há limitação produzida pela dor e pela inflamação ${ }^{2-4}$.

A educação dos pacientes visa a aumentar o seu conhecimento acerca de sua própria doença, além de orientar a implementação de técnicas de proteção articular (PA) e conservação de energia (CE), tendo como objetivo a minimização de perdas funcionais que acompanham a $\mathrm{AR}^{2,5}$.

De acordo com Noordhoek e Loschiavo ${ }^{6}$, o método de intervenção grupal é adequado para pacientes com doenças crônicas, pois neste tipo de intervenção são trabalhadas também trocas de experiências individuais com a doença, fazendo com que os indivíduos assumam um papel mais ativo, na medida em que mobiliza a participação e controle diante dos sintomas da doença, atuando, assim, diretamente, no incremento da qualidade de vida e prevenção dos agravos.

Grupos de pacientes com AR que objetivam à orientação acerca da natureza da sua doença e tratamento, compartilhamento de experiência, e orientações sobre medidas de PA e CE têm boa aceitação e associam-se a melhora do conhecimento, porém os resultados são controversos em relação à melhora da função articular, do desempenho ocupacional, na aquisição de maior independência, na redução da dor e na melhora da qualidade de vida ${ }^{1-3,5,6}$.

\section{OBJETIVO}

Avaliar o efeito da participação em grupos de orientação a pacientes com AR na sua autopercepção de dor e qualidade de vida.

\section{PACIENTES E MÉTODOS}

Esse trabalho é o projeto piloto de um ensaio clínico de intervenção terapêutica aberto, registrado no Registro
Brasileiro de Ensaios Clínicos (ReBEC) sob o número RBR-4rj39m.

Pacientes com diagnóstico de AR segundo Critérios de Classificação American College of Rheumatology (ACR)/ European League Against Rheumatism (EULAR) $2010^{7}$, maiores de 18 anos de idade, acompanhados ambulatorialmente no Hospital das Clínicas da UFMG, foram convidados a participar dos grupos de orientação.

Os pacientes foram atendidos em encontros semanais, em grupos de oito a doze pacientes por sessão, com duração duas horas/semana, durante 12 semanas.

O estudo foi realizado entre agosto de 2018 e julho de 2019. Foram realizadas ações educativas que envolveram exposições teóricas projetadas (aulas informativas acerca da doença, seu tratamento, medidas de PA e CE, exercícios terapêuticos) e práticas com orientações coletivas e individuais direcionadas aos participantes do grupo, além do compartilhamento de vivências entre os participantes.

Os profissionais envolvidos nos grupos foram acadêmicos e profissionais de medicina (Reumatologia) e terapia ocupacional.

Este estudo foi aprovado no Comitê de Ética em Pesquisa da Universidade federal de Minas Gerais em 03 de julho de 2018 (CAAE: 86780648.4.0000.5149).

Todos os pacientes leram, entenderam e assinaram o Termo de Consentimento Livre e Esclarecido (TCLE). Aqueles que não concluíram sua participação nos grupos ou não foram capazes de entender e assinar o TCLE ou entender e responder aos questionários de avaliação foram excluídos.

A percepção da dor relatada pelo paciente em escala visual analógica (EVA dor) e qualidade de vida avaliada pelo sistema descritivo em três níveis de cinco dimensões do EuroQol e sua escala visual analógica (EQ-5D-3L e EQ-VAS) foram avaliadas no primeiro e último encontro do grupo ${ }^{8-11}$.

$\mathrm{Na}$ EVA dor os pacientes foram instruídos a fazer uma única marca vertical em uma linha de $10 \mathrm{~cm}$ orientada horizontalmente, rotulada em seus extremos como "sem dor nenhuma" (à esquerda) e "a pior que já sentiu" (à direita) referente à dor sentida pelo paciente na última semana ("marque na linha abaixo como esteve a dor da sua artrite na última semana"). A distância entre a extremidade esquerda e a marca do paciente foi medida em milímetros para análise no presente estudo.

O EQ-5D-3L descreve a saúde geral no dia da aplicação ("hoje") em cinco dimensões: mobilidade, cuidados pessoais, atividades habituais, dor/mal-estar, ansiedade/ depressão ${ }^{8}$. Cada dimensão tem três níveis, graduados de um a três: não tem problemas, tem alguns problemas, tem problemas importantes/é incapaz de realizar as atividades. Ele é descrito conforme a graduação em cada nível e 
varia de 11111 (o melhor estado de saúde geral) a 33333 (o pior estado de saúde geral). Ele é convertido em um índice que atribui um valor numérico a cada nível de saúde em cada dimensão e foi calculado a partir de estudo brasileiro. Nesse trabalho, tal índice variou de 1,0 (o melhor estado de saúde) a -0,223 (o pior estado de saúde) ${ }^{12}$.

A EQ-VAS é uma EVA orientada verticalmente, graduada de zero (o pior estado de saúde imaginável) a 100 (o melhor estado de saúde imaginável), em que o paciente registra com uma única marca horizontal seu estado de saúde no dia da aplicação ("hoje").

Foi utilizada a versão em Português do Questionário de Saúde para aplicação presencial fornecido pelo grupo EuroQol ${ }^{10,11}$.

Deformidades em mãos pela AR foram definidas como a presença de dedos em pescoço de cisne ou botoeira, desvio ulnar dos dedos, mãos em dorso de camelo ou polegares em Z. Sua presença foi avaliada no primeiro encontro dos grupos. ${ }^{4}$

Foi feita revisão dos prontuários médicos dos pacientes para coleta de informações acerca das características da AR (duração, alterações sorológicas e radiográficas) atividade da doença (medida pelo índice composto de atividade da doença Disease Activity Score usando contagem de 28 articulações dolorosas e edemaciadas e proteína $\mathrm{C}$ reativa sérica - DAS28-PCR) e do seu tratamento.

\section{Estatísticas}

Foi utilizando o programa SPSS versão 23.0 (SPSS Inc., Chicago, IL, USA).

Efetuou-se a análise descritiva das características demográficas e da AR. As variáveis numéricas foram avaliadas quanto à normalidade pelo teste de Shapiro-Wilk. São mostradas em médias e desvio-padrão (variáveis numéricas de distribuição normal), medianas e intervalo interquartil (variáveis numéricas de distribuição não normal) e valores absolutos e frequências (variáveis categóricas). $\mathrm{Na}$ análise de correlações e associações das características basais dos pacientes, foram usados os testes de correlação de Pearson (variáveis numéricas de distribuição normal) ou Spearman (variáveis numéricas de distribuição não normal), o Teste T de Student (comparação de médias) e o teste de MannWhitney (variáveis numéricas de distribuição não normal). Para avaliação dos efeitos da intervenção nos parâmetros avaliados, utilizaram-se os testes t-pareado (variáveis numéricas de distribuição normal) e de Wilcoxon (variáveis numéricas de distribuição não-normal). $\mathrm{O}$ valor de $\mathrm{p}<0,05$ foi considerado significativo.

\section{RESULTADOS}

Esse estudo piloto descreve os resultados dos primeiros 15 pacientes com AR incluídos nos grupos de orientação. As características dos pacientes são mostradas na Tabela 1. Foram incluídos no estudo os pacientes que obtiveram frequência de participação dos encontros superior a $80 \%$. Quatorze (93\%) deles eram do gênero feminino. Os pacientes apresentaram idade entre 46 a 75 anos e tempo de duração da AR de um a 37 anos. Essa população é acompanhada através do sistema público de saúde e se caracterizou por renda familiar mensal baixa, que variou entre um e seis salários mínimos, além de baixo nível de escolaridade (12 pacientes apresentaram menos de oito anos de estudo e três completaram 11 anos de estudo).

Tabela 1 - Características dos pacientes

\begin{tabular}{lc}
\hline Variável & $\mathbf{N}$ \\
\hline Gênero Feminino* $^{*}$ & $14(93 \%)$ \\
Cor branca* $^{*}$ & $3(20 \%)$ \\
Idade (anos) $^{* *}$ & $61,27(9,25)$ \\
Renda familiar mensal (SM) $^{\&}$ & $2,0(1,26)$ \\
Tempo de AR (anos) $^{* *}$ & $19,93(10,31)$ \\
FR positivo $^{1}$ & $9(69 \%)$ \\
ACCP positivo $^{2}$ & $5(62,5 \%)$ \\
Deformidades articulares em mãos*** $^{*}$ & $13(87 \%)$ \\
Alterações radiográficas em mãos & \\
DAS28-PCR & $11(100 \%)$ \\
Uso de PDN & $3,04(1,7)$ \\
Uso de DMCD & $3(20 \%)$ \\
\hline
\end{tabular}

Legenda: AR: artrite reumatoide. FR: fator reumatoide. ACCP: antiCCP. SM: salários mínimos (no período do estudo, o salário mínimo correspondia a aproximadamente US\$250,00). DAS28-PCR: Disease Activity Score. PDN: prednisona. DMCD: droga modificadora do curso da doença. ***Deformidades foram definidas como a presença de dedos em pescoço de cisne ou botoeira, desvio ulnar dos dedos, mãos em dorso de camelo ou polegares em Z. ${ }^{1}$ Resultados para o fator reumatoide foram disponíveis para 13 pacientes; ${ }^{2} \mathrm{O}$ resultado do ACCP foi disponível para 8 pacientes; ${ }^{3}$ Descrição de radiografias das mãos estavam relatadas nos prontuários de 11 pacientes. A presença de erosões ou rarefação justa-articular foi considerada sugestiva da AR. *Valor absoluto (\%). **Média (DP). ${ }^{\&}$ Mediana (Intervalo interquartil)

Fonte: Borges LCD, 2020.

Nenhum paciente mantinha atividade laborativa remunerada formal. Treze (87\%) deles já haviam se aposentado e dois estavam desempregados. Dos pacientes aposentados, 
$12(92 \%)$ haviam se aposentado por incapacidade associada à $\mathrm{AR}$ e apenas um se aposentou pelo tempo regulamentar de trabalho e contribuição previdenciária.

Catorze pacientes $(93 \%)$ recebiam tratamento com MMCD, 12 (80\%) usavam MMCD sintéticos convencionais (metotrexato 9; leflunomida 4) e quatro (27\%) usavam MMCD biológico (adalimumabe: 1, tocilizumabe: 2, abatacepte: 1).

Durante o estudo não houve alteração no tratamento medicamentoso da AR.

Conforme avaliação da atividade da AR pelo DAS28PCR (Disease Activity Score) que usa a contagem de 28 articulações dolorosas e edemaciadas, a EVA global do paciente e a proteína $C$ reativa), os pacientes encontravamse inativos/em remissão, ou com atividade baixa, moderada ou alta de sua artrite, mas apresentavam, em média, baixa atividade da doença ${ }^{13}$.
Quando do início da participação dos pacientes nos grupos de pacientes, houve correlação significativa entre a EVA dor e um pior qualidade de vida, avaliada pelo índice numérico do EQ-5D-3L ( $r=-0,549 ; \mathrm{p}=0,05)$ e pelo EQVAS $(r=-0,765 ; p=0,002)$. A idade, o tempo de duração da $\mathrm{AR}$, a aposentadoria por incapacidade, o uso de DMCD e a presença de deformidades em mãos não se associaram aos parâmetros avaliados.

Após participação nos grupos, houve tendência à redução da dor e melhora da qualidade de vida avaliada pelo índice do EQ-5D-3L e pela EQVAS, sem significância estatística. Apesar do pequeno número de pacientes incluídos neste estudo piloto, foi observada melhora significativa em relação ao domínio do EQ-5D-3L referente aos cuidados pessoais (Tabela 2).

Tabela 2 - Variáveis comparativas entre o início e final dos grupos

\begin{tabular}{lccc}
\hline Variável & T0 & T1 & Valor P \\
\hline EVA dor $(\mathrm{mm})^{* *}$ & $54,58(30,26)$ & $42,5(30,19)$ & 0,13 \\
EQ-5D-3L** & $0,620(0,174)$ & $0,631(0,235)$ & 0,28 \\
EQ5D Mobilidade\& $^{*}$ EQ5D Cuidados pessoais $^{\&}$ & $2,0(1,0)$ & $2,0(1,0)$ & 1,0 \\
EQ5D Atividades habituais $^{\&}$ & $2,0(1,0)$ & $1,0(1,0)$ & $\mathbf{0 , 0 4 6 *}$ \\
EQ5D Dor/mal-estar $^{\&}$ & $2,0(1,0)$ & $2,0(1,0)$ & 0,32 \\
EQ5D Ansiedade/depressão\& & $2,0(1,0)$ & $2,0(1,0)$ & 0,56 \\
EQ5D VAS** & $2,0(1,0)$ & $2,0(1,0)$ & 1,0 \\
\hline
\end{tabular}

EVA: Escala visual analógica. 'EQ-5D-3L: índice derivado do EuroQol 5D-3L. EQ5DVAS: escala visual analógica do EuroQol. EQ5D Mobilidade, cuidados pessoais, atividades habituais, dor/mal-estar e ansiedade/depressão: domínios do EuroQol. ** Média (Desvio-padrão). ${ }^{*}$ Mediana (Intervalo interquartil) ${ }^{*} \mathrm{p}<0,05$ Fonte: Borges LCD, 2020.

As medidas educacionais e de terapia ocupacional não se associaram a piora da atividade da AR (mediana e intervalo interquartil) do DAS 28-PCR inicial 3,04 (1,71) vs final $2,56(3,05), \mathrm{p}=0,87)$.

Durante sua participação nos grupos, todos os pacientes relataram a adoção de alguma das medidas de $\mathrm{CE}$ ou PA em sua rotina diária e relatavam estar satisfeitos com as atividades desenvolvidas.

\section{DISCUSSÃO}

Apesar deste estudo tratar-se de um projeto piloto que incluiu apenas 15 pacientes com AR e, portanto, seus resultados exigirem cautela na interpretação, observouse melhora não significativa na autopercepção de dor e qualidade de vida pelos pacientes, além de uma melhora significativa nos cuidados pessoais dos mesmos, avaliado pelo domínio do questionário EQ-5D-3L. Houve, ainda, relato de boa aceitação e adoção de algumas das medidas de PA e CE sugeridas. Os pacientes relataram boa satisfação com sua participação nos grupos.

A participação de pacientes com AR em grupos de orientação que tratam da natureza de sua doença e tratamento além de fornecer orientações acerca de PA e CE e compartilhamento de vivências visa ao maior conhecimento deles sobre sua doença, de modo que possam assumir um papel mais ativo no processo de seu tratamento, com o objetivo de manter a integridade articular, reduzir a dor e contribuir para uma melhor percepção de qualidade de vida por esses indivíduos ${ }^{6}$.

As pacientes eram do sexo feminino, com média de idade e aspectos sócio demográficas semelhantes aos pacientes 
acompanhados em serviço público de saúde brasileiro. A alta frequência de pacientes aposentados pela $\mathrm{AR}$ reflete sua importância como causa frequente de afastamento do trabalho e incapacidade. As características clínicas, sorológicas, terapêuticas e ocupacionais da população do presente estudo também se assemelham às de populações de outros trabalhos $3,13,15,20,21$.

Neste estudo, foram incluídos pacientes com AR de longa evolução, que já apresentavam deformidades e alterações radiográficas pela doença, o que pode justificar os altos índices de dor e a baixa qualidade de vida apresentados pelos pacientes e a ausência de melhora significativa destes parâmetros pelas intervenções educativas realizadas durante os grupos de pacientes ${ }^{16,17}$. Além disto, a associação entre a dor e uma pior qualidade de vida corrobora achados prévios ${ }^{18}$.

Neste estudo houve uma tendência à melhora da dor, embora sem significância estatística. Os fatos deste se tratar de um projeto piloto, que incluiu pequeno número de pacientes, que já apresentavam AR de longa duração e com deformidades articulares estabelecidas e foram acompanhados nos grupos de pacientes por um período relativamente curto podem justificar a ausência de benefício significativo da participação dos grupos de pacientes na sua dor. O impacto da participação de pacientes com AR em grupos de orientação sobre a dor mostra resultados conflitantes em diferentes estudos ${ }^{2,21-23}$.

No Brasil, esse é o primeiro projeto que avaliou o impacto da participação de pacientes com AR em projeto de educação sobre a qualidade de vida utilizando o EQ-5D-3L e o EQ-VAS. A melhora da autopercepção dos pacientes acerca dos seus cuidados pessoais corrobora um impacto positivo da educação e do tratamento não medicamentoso na qualidade de vida destes pacientes ${ }^{22,23}$. Mesmo pacientes com AR de longa evolução, com deformidades articulares e limitações funcionais, que participaram dos grupos de orientação aos pacientes deste estudo, foram beneficiados com as orientações obtidas durante sua participação. Com essa estratégia eles aprenderam novas formas de lidar com suas limitações e desenvolveram maior habilidade e independência no desempenho de atividades de autocuidado.

$\mathrm{O}$ alto grau de satisfação dos pacientes participantes dos grupos de orientação corrobora resultados de outros estudos, despertando maior interesse por essa modalidade de atendimento, melhor capacidade de enfrentamento de sua doença, e aumento da socialização ${ }^{20,23}$.

\section{CONCLUSÃO}

A participação de pacientes com AR em grupos de orientação, que tratam da natureza de sua doença e tratamento e fornecem orientações e atividades terapêuticas ocupacionais acerca de PA e CE, visa ao maior conhecimento dos mesmos sobre sua doença, de modo que possam assumir um papel mais ativo no processo de seu tratamento e na melhora dos cuidados pessoais. E dessa forma, manter a integridade articular e reduzir a dor. Ao fim do estudo, concluiu-se que houve uma melhora na autopercepção da dor e da qualidade de vida por estes indivíduos, contudo, não houve diferença estatística significativa. Deve-se salientar, no entanto, que o reduzido número de pacientes incluídos, apenas 15 pacientes com AR, por se tratar de um estudo piloto, com características de longo tempo de evolução de doença, presença frequente de alterações radiográficas e deformidades articulares, alto índice de dor e baixa qualidade de vida basais podem ter impactado nesse achado estatístico. A continuidade dos grupos de orientação aos pacientes com AR e a inclusão de mais pacientes poderão, portanto, esclarecer melhor o impacto da educação na saúde desses indivíduos e nas suas percepções de dor e de qualidade de vida.

Agradecimentos: Agradecemos ao Grupo EuroQol pela autorização e orientações para o uso do questionário Euro-QOL-5D-3L, aos coordenadores dos ambulatórios de AR do HC-UFMG, Dra. Maria Raquel da Costa Pinto, Dra. Maria Fernanda Brandão de Resende Guimarães, Dr. Ricardo Cruz Lage, à equipe de Terapia Ocupacional da Unidade Multiprofissional e Reabilitação do Hospital das Clínicas e aos acadêmicos de Medicina e Terapia Ocupacional que participaram da condução dos grupos dos pacientes com artrite reumatoide. Os respectivos autores, Borges LCD, Chaves LA, Andrade JÁ, Silva LC, Marcelino ALFO, Chaves RA, Kakehasi AM, Calderaro DC contribuíram igualmente em todas as funções da elaboração do artigo.

\section{REFERÊNCIAS}

1. Ekelman BA, Hooker L, Davis A, Klan J, Newburg D, Detwiler K, et al. Occupational therapy interventions for adults with rheumatoid arthritis: an appraisal of the evidence. Occup Ther Health Care. 2014;28(4):347-61. https://doi.org/ 10.3109/07380577.2014.919687.
2. Torquetti A, Campos TS, Noordhoek J, Cassiano JG. Programas de proteção articular para indivíduos com artrite reumatoide: uma revisão da literatura. Rev Ter Ocup Univ Sao Paulo. 2008;19(2):76-84. https://doi.org/10.11606/issn.22386149.v19i2p76-84. 
3. Macedo AM, Oakley SP, Panayi GS, Kirkham BW. Functional and work outcomes improve in patients with rheumatoid arthritis who receive targeted, comprehensive occupational therapy. Arthritis Rheum (Arthritis Care Res). 2009;61(11):1522-30. https://doi.org/10.1002/art.24563.

4. Goeldner I, Skare TL, Razão ITM, Utiyama, SRR. Artrite reumatoide: uma visão atual. J Bras Patol Med Lab. 2011;47(5):495-503. https://doi.org/10.1590/S167624442011000500002 .

5. Almeida PHTQ, Pontes TB, Matheus JPC, Muniz LF, Mota LMH. Occupational therapy in rheumatoid arthritis: what rheumatologists need to know? Rev Bras Reumatol. 2015,55(3):272-80. https://doi.org/10.1016/j.rbr.2014.07.008.

6. Noordhoek J, Loschiavo FQ. Intervenção da terapia ocupacional no tratamento de indivíduos com doenças reumáticas utilizando a abordagem da proteção articular. Rev Bras Reumatol. 2005;45(4):242-4. https://doi.org/10.1590/ S0482-50042005000400008.

7. Aletaha D, Neogi T, Silman A, Funovits J, Felson D, Bingham $\mathrm{C}$, et al. The 2010 rheumatoid arthritis classification criteria: an American College of Rheumatology / European League Against Rheumatism collaborative initiative. Arthritis Rheum. 2010;62(9):2569-81. https://doi.org/10.1002/art.27584.

8. Rabin R, Charro F. EQ-5D: a measure of health status from the EuroQol Group. Ann Med. 2001;33(5):337-43. https://doi.org/10.3109/07853890109002087.

9. Martinez JE, Grassi DC, Marques LG. Análise da aplicabilidade de três instrumentos de avaliação de dor em distintas unidades de atendimento: ambulatório, enfermaria e urgência. Rev Bras Reumatol. 2011;51(4):304-8. https://doi.org/10.1590/S048250042011000400002 .

10. Ferreira PL, Ferreira LN, Pereira LN. Contributos para a validação da versão Portuguesa do EQ-5D. Acta Med Port. 2013;26(6): 664-75. Disponível em: http://www.actamedicaportuguesa.com/ revista/index.php/amp/article/viewFile/1317/3807.

11. Brooks R. EuroQol: the current state of play. Health Policy. 1996;37(1):53-72. https://doi.org/10.1016/01688510(96)00822-6.

12. Andrade MV, Noronha K, MPhil PK, Maia AC, Menezes RM, Reis CB, et al. Societal Preferences for EQ-5D health states from a Brazilian Population Survey. Value Health Reg Issues. 2013;2(3):405-12. https://doi.org/10.1016/j.vhri.2013.01.009.

13. Wells G, Becker J-C, Teng J, Dougados M, Schiff M, Smolen J, Aletaha D, van Riel PLCM. Validation of the 28-joint Disease Activity Score (DAS28) and European League Against Rheumatism response criteria based on C-reactive protein against disease progression in patients with rheumatoid arthritis, and comparison with the DAS28 based on erythrocyte sedimentation rate. Ann Rheum Dis. 2009;68:954-60. http://dx.doi.org/10.1136/ard.2007.084459.
14. da Rocha Castelar-Pinheiro G, Vargas-Santos AB, de Albuquerque CP, Bértolo MB, Júnior PL, Giorgi RDN, Radominski SC, et al. The REAL study: a nationwide prospective study of rheumatoid arthritis in Brazil. Adv Rheumatol. 2018;58(1):9. https://doi.org/10.1186/s42358-018-0017-9.

15. Santos AB, Martins AR, Leitão JMSR, Barros AGTS, Cavalcante GL, Menese CES, et al. Perfil fármaco epidemiológico de portadores de artrite reumatoide. Rev Eletrônica Acervo Saúde. 2018;12:e213. https://doi.org/10.25248/reas.e213.2018.

16. Hoshi D, Tanaka E, Igarashi A, Inoue E, Kobayashi A, Sugimoto N, et al. Profiles of EQ-5D utility scores in the daily practice of Japanese patients with rheumatoid arthritis; Analysis of the IORRA database. Mod Rheumatol. 2018;26(1):40-5. https://doi.org/10.3109/14397595.2015.1059983.

17. Kim H-L, Kim D, Jang EJ, Lee M-Y, Song HJ, Park S-Y, et al. Mapping health assessment questionnaire disability index (HAQ-DI) score, pain visual analog scale (VAS), and disease activity score in 28 joints (DAS28) onto the EuroQol-5D (EQ-5D) utility score with the KORean Observational study Network for Arthritis (KORONA) registry data. Rheumatol Int. 2016;36(4):505-13. https://doi.org/10.1007/s00296-016-3427-1.

18. Katchamart W, Narongroeknawin P, Chanapai W, Thaweeratthakul P. Health-related quality of life in patients with rheumatoid arthritis. BMC Rheumatol. 2019;3:34. https://doi.org/10.1186/s41927-019-0080-9.

19. Gomes CMS, Coutinho GC, Miyamoto ST. Efeitos do programa de educação em pacientes com artrite reumatoide do Serviço de Reumatologia do Hospital Universitário Cassiano Antônio de Moraes (HUCAM) - projeto piloto. Rev Ter Ocup Univ Sao Paulo. 2014;24(3):250-8. https://doi.org/10.11606/ issn.2238-6149.v24i3p250-258.

20. Lindroth Y, Brattstrom M, Bellman I, Ekestaf G, Olofsson Y, Strömbeck B, et al. A problem-based education program for patients with rheumatoid arthritis: evaluation after three and twelve months. Arthritis Care Res. 1997;10(5):325-32. https://doi.org/10.1002/art.1790100507.

21. Hammond A, Lincoln N, Sutcliffe L. A crossover trial evaluating an educational-behavioural joint protection programme for people with rheumatoid arthritis. Patient Educ Couns. 1999;37(1):19-32. https://doi.org/10.1016/ S0738-3991(98)00093-7.

22. Hammond A, Jeffreson $\mathrm{P}$, Jones $\mathrm{N}$, Gallagher J, Jones T. Clinical Applicability of an Educational-Behavioural Joint Protection Programme for People with Rheumatoid Arthritis. Br J Occup Ther. 2002;65(9):405-12. https://doi.org/10.1177/030802260206500903.

23. Carvalho MGR, Noordhoek J, Silva MCO. Grupo de orientação a indivíduos acometidos por doenças reumáticas: espaço educativo e terapêutico. Rev Bras Reumatol. 2006;46(2): 134-6. https://doi.org/10.1590/S0482-50042006000200008. 